\title{
ISG15 and ISGylation: Emergence in the cytoskeleton dynamic and tumor microenvironment
}

\author{
Angeles C. TECALCO-CRUZ* \\ Posgrado en Ciencias Genómicas, Universidad Autónoma de la Ciudad de México (UACM), Ciudad de México, 03300, México
}

Key words: Tumor microenvironment, ISG15, ISGylation, Cytoskeleton dyn

\begin{abstract}
Cytoskeletal remodeling affects the shape, adhesion, and motility of cells. Cytoskeletal dynamics are modulated by matrix proteins, integrins, and several cytokines in the tumor microenvironment. In this scenario, signaling is activated by integrins and interferons, which can induce ISG15 gene expression. This gene encodes a ubiquitin-like protein that functions as a protein modifier via the ISGylation system. Furthermore, non-conjugated ISG15 acts as a cytokine-like protein. In this viewpoint, the interplay between free ISG15, protein ISGylation, and cytoskeletal dynamics in the tumor microenvironment is discussed.
\end{abstract}

\section{Introduction}

A tumor is not isolated but surrounded by an entire microenvironment with which it interacts, maintaining reciprocal communication. Different elements comprise the tumor microenvironment, such as different cell types, including vascular cells, cancer-associated fibroblasts (CAFs), tumor-associated macrophages (TAMs), and other immune cells. The tumor microenvironment contains various molecules implicated in cellular communication, such as extracellular matrix (ECM) proteins, integrins, cytokines, and several molecules contained in extracellular vesicles (EVs) (Zhang et al., 2021). In the tumor microenvironment, biochemical factors and biomechanical stimuli are generated by ECM. Both elements trigger signaling that activates cytoskeleton remodeling, influencing tumor cell decisions by regulating cell morphology, spreading, migration, metastasis, and invasion (Li and Wang, 2020).

Integrins are heterodimers composed of $\alpha$ and $\beta$ chains that form transmembrane receptors to recognize various surface molecules of other cells, EMC proteins, and specific soluble proteins. Integrins mediate cell adhesion to the ECM and act as mechanosensors because integrins can sense mechanical forces in the cells and their tumor microenvironment. Hence, integrins transduce signaling from the extracellular to the intracellular space and vice versa, presenting in all the cells of the surrounding tumor microenvironment (Li and Wang, 2020;

*Address correspondence to: Angeles C. Tecalco-Cruz, angeles.tecalco@uacm.edu.mx

Received: 30 June 2021; Accepted: 09 August 2021
Tang et al., 2021). Several types of integrins are differentially expressed in diverse cell types. For example, cells of the immune system can present integrins $\beta 2$ and $\beta 7$, while leukocytes and lymphocytes display LFA-1 ( $\alpha \mathrm{L} \beta 2, \mathrm{CD} 11 \mathrm{a} /$ CD18). In summary, integrins represent a system that senses and manages responses to modifications of the tumor microenvironment (DiPersio and Longmate, 2017).

Furthermore, immune cells contribute to the tumor environment by secreting and recognizing cytokines (LabaniMotlagh et al., 2020). The primary role of immune system cells in the tumor microenvironment is to target and eliminate tumor cells. However, tumor cells can acquire immune system evasion ability (Lei et al., 2020). In all these scenarios, ISG15 is emerging as a critical element in cytoskeleton dynamics and the tumor microenvironment, as discussed below.

\section{Interferon-Stimulated Gene 15 (ISG15)}

Interferons (IFNs) are a family of proteins having antiviral, antiproliferative, and proapoptotic activities through the activation of a JAK-STAT system that leads to the expression of interferon-stimulated genes (ISGs) (Schoggins, 2019; Jorgovanovic et al., 2020). Many ISGs encode proteins that can promote or inhibit tumor growth. ISG15 is one of the most enigmatic interferon-stimulated genes because of its expression, modulation, and function. For instance, ISG15 is expressed in vertebrates and encodes a ubiquitinlike protein. ISG15 protein can be detected in two forms: conjugated and free.

a) Conjugated ISG15: ISG15 is covalently associated with lysine residues of its target proteins. The covalent interaction 
between ISG15 and its target proteins occurs via a catalytic process known as ISGylation, which is mediated by an E1activation, E2-conjugation, and E3-ligase enzymes for ISG15. Hence, ISGylation is a posttranslational modification, and can be reverted by the USP18 enzyme, which removes ISG15 from its target proteins (Tecalco-Cruz, 2020) (Fig. 1).

b) Free ISG15: ISG15 is not covalently bound to other proteins. Moreover, free ISG15 can be detected as a free protein localized intracellularly and/or extracellularly.

ISGylation has garnered increasing attention in recent years, based on the fact that ISGylation can increase or reduce protein stability or protein-protein interactions. ISGylation is detected in the nucleus and cytoplasm, but a limited number of targets have been identified. For example, cytoskeleton-associated proteins such as IQGAP1 (Cerikan et al., 2016); and nuclear proteins with activity of transcription factors, such as STAT1 and IRF3, are modified by ISGylation (Shi et al., 2010; Ganesan et al., 2016).

Instead, free ISG15 acts as a cytokine because it is produced and secreted from immune system cells, is recognized by the LFA-1 integrin-type receptor and has pleiotropic activities (Swaim et al., 2017, 2020). Thus, the multifunctionality of ISG15 associated with its activity as a cytokine, and as a protein modifier exclusive of vertebrate organisms, distinguishes it from other ISGs.

\section{ISG15 Emerging in the Tumor Microenvironment Stage}

ISG15 is upregulated in most of the cancer types and is associated with a pro-tumor action. Nevertheless, the proteins that are ISGylated in these cancer types are not completely defined. Instead, it has been found that free ISG15 is secreted by cancer cells such as melanoma, esophageal squamous cell carcinoma (ESCC), pancreatic ductal adenocarcinoma (PDAC), and nasopharyngeal carcinoma (NPC) cells (Padovan et al., 2002; Sainz et al., 2014; Chen et al., 2016; Yuan et al., 2018). In PDAC and NPC, ISG15 secretion enhances the cancer stem cell phenotype. Tumor-associated macrophages (TAMs) in PDAC and NPC also secrete ISG15, which is associated with poor patient survival (Sainz et al., 2014; Chen et al., 2016). The presence of ISG15 in the tumor microenvironment promotes the induction of macrophages that exhibit an M2-like phenotype, cell migration, and tumor progression (Chen et al., 2020).

Moreover, it has been demonstrated that free ISG15 is secreted by cells such as monocytes and lymphocytes and is then recognized by the LFA-1 integrin receptor present in natural killer (NK) cells, inducing the secretion of cytokines such as IL-10 and IFN- $\gamma$ (Swaim et al., 2017). The communication between immune cells and tumor cells through extracellular ISG15 may play central role in the tumor microenvironment.

In addition, EVs are released from different cell types and transport diverse molecules. Consequently, EVs are important for cell communication in the tumor microenvironment and modulate several actions such as angiogenesis, cell differentiation, metabolic reprogramming, and treatment resistance (Zhang et al., 2021). As a regulation point, multivesicular bodies (MVB) can be degraded or secreted via exosomal EVs. Interestingly, ISGylation of MVB proteins such as TSG101 is related to the inhibition of exosomal EV secretion, promoting the lysosomal degradation of MVB (Villarroya-Beltri et al., 2016). Thus, ISGylation may also modulate the communication of the tumor microenvironment cells via removal of EVs.

However, it is important to mention that antitumor activity of ISG15 has been reported in ovarian, cervical, and blood cancer cells (Mao et al., 2016; Zhou et al., 2017; Yeung et al., 2018). Although the mechanisms that define the pro and antitumor effects of ISG15 are not clear, this fact suggests a complex crosstalk between ISG15-associated mechanisms and several oncogenic pathways that are dependent on cell type. Accordingly, the molecular target partner for ISGylation may differ and/or be sequential to other modifications or signals in different cancer types.

\section{ISG15 and ISGylation in the Cytoskeleton Dynamic}

Few ISGylation targets have been identified, and some of them include NMIIA, IQGAP1, and Filamin B, proteins linked to the actin cytoskeleton (Desai et al., 2006; Jeon et al., 2009; Lee et al., 2010).

For example, IFN- $\gamma$ has been reported to induce changes in the morphology of breast cancer cells, which seems to be related to the induction of ISGylation, promoting changes in the cytoskeleton and the distribution of F-actin and stress fibers (Tecalco-Cruz et al., 2019).

The actomyosin complex is the association between NMIIA and F-actin, and is essential for the organization of the cytoskeleton. Interestingly, IFN- $\gamma$ induces NMIIA ISGylation, modulating the interactions of NMIIA with some cytoskeletal proteins. Also, NMIIA ISGylation could be a key mechanism during cell spreading, a process essential for cell movement

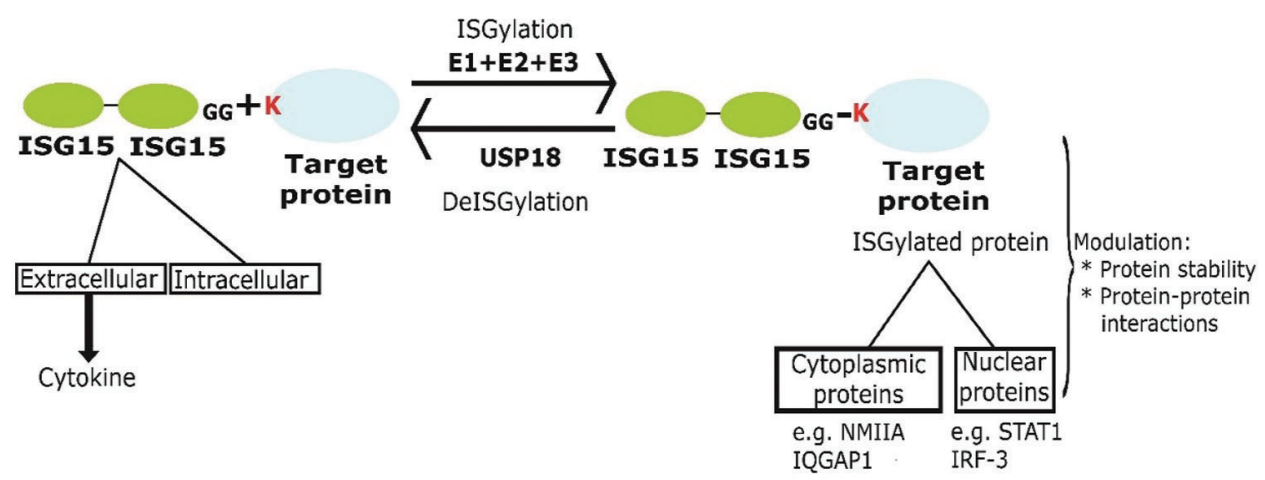

FIGURE 1. Free ISG15 and ISGylation system. Subcellular distribution and functions of free or conjugated ISG15. 
and migration (Cruz-Ramos et al., 2019). Hence, in the attachment of the cells at the substrate, as well as the detachment, cell spreading is an initial step, and myosin proteins modulate this event (Betapudi et al., 2006; Cai et al., 2006; Betapudi, 2010). Particularly, it has been suggested that the ISGylation of NMIIA can occur in the spreading of breast cancer cells induced by fibronectin, an extracellular matrix protein (Cruz-Ramos et al., 2019). In addition, fibronectinbinding integrins of $\alpha v$ and $\beta 1$-class activate MRTF-A/SRF transcription factors to induce ISG15 expression, which has been associated with IQGAP1 ISGylation in MDA-MB-231 breast cancer cells (Cerikan et al., 2016; Hermann et al., 2016).

Therefore, extracellular matrix proteins such as fibronectin have an effect on ISG15 expression, increasing the ISGylation of cytoskeleton proteins such as NMIIA and IQGAP1, modulating cytoskeletal organization, cell morphology, and spreading required for cell migration. Thus, the regulation of cytoskeleton by ISGylation is connected to factors or signals from the tumor microenvironment.

\section{ISG15 and ISGylation in the Breast Cancer Context}

In vitro and in vivo studies have shown the implications of ISG15 in a breast cancer context by using RNA interference specific for ISG15. In vitro, a reduction in proliferation and migration when the levels of ISG15 are decreased has been observed. Also, a reversion of epithelial-mesenchymal transition of breast cancer cells occurs when the levels of ISGylation are reduced. A similar phenotype was observed using RNA interference to reduce the expression of UBCH8 (conjugation enzyme for ISGylation) (Desai et al., 2012; Burks et al., 2014). These results suggest the pro-tumor potential of ISGylation in breast cancer cell lines.

In contrast, in vivo mouse models, xenotransplantation of breast cancer cells with a decrease in ISG15 expression generated an increase in tumor incidence and size, suggesting an antitumor role for ISG15 (Burks et al., 2015, 2019). These differences in pro-and antitumor activities of ISG15 in vitro (2D cell culture) compared with in vivo (xenotransplantation in athymic mice) models may be related to the lack or existence of a tumor microenvironment, respectively. Thus, factors from the tumor environment may trigger signaling with the ability to regulate ISG15 actions.

These studies have analyzed the actions of ISG15 by reducing its expression via RNA interference but not discern between the implications of the free and conjugated ISG15 forms. Nonetheless, an in vivo study showed that the injection of exogenous free ISG15 close to breast cancer cell xenotransplantation significantly reduced tumor development, suggesting its antitumor function by favoring NK cell infiltration into the tumor (Burks et al., 2015). Moreover, free ISG15 is a potent adjuvant for cytotoxic $\mathrm{T}$ lymphocyte (CTL) responses through an NK cell-dependent pathway (Iglesias-Guimarais et al., 2020). These data indicate that the administration of ISG15 in the tumor microenvironment may have an antitumor function.

\section{Potential Implications of ISG15 as a Factor of Tumor Microenvironment Remodeling}

Tumor cells can sense the rigidity and extracellular composition of the surrounding microenvironment, inducing changes in the cytoskeletal organization of tumor cells to adapt or respond to external circumstances. According to previous reports, various elements of the microenvironment, including integrins and IFNs, can induce the expression of ISG15, generating various possible routes to modulate the cytoskeleton dynamic and tumor microenvironment (Sainz et al., 2014; Hermann et al., 2016) (Fig. 2).

Thus, it is possible to propose:

1) ISGylation modulates the cytoskeleton dynamics and exosome secretions, repercuting in the cell behavior, cell signaling and cell-cell communication in the microenvironment.

2) The proteome modifications by ISGylation of cells that integrate the tumor microenvironment are incompletely defined, but ISGylation may determine specific cell responses.

3) Transcriptional and epigenetic regulators may also be modified by ISGylation, modulating the transcriptome, and generating a gene expression signature that directs cell fate.

4) Free ISG15 stimulates cytokine production in some cells. For instance, ISG15 induces the production of IFN- $\gamma$ in NK cells, suggesting that free ISG15 affects the cell secretome of the tumor microenvironment.

5) Free ISG15 can also recruit cells from the immune system to fight against tumors. Therefore, free ISG15 may improve immunotherapy in several cancer types.

6) Free ISG15 can also mediate a signaling that suppresses the immune response in some cancer types under specific conditions.

Although in general, it would be assumed that free ISG15 and ISGylation have antitumor and tumor-promoting functions, respectively, the type of tumor and the composition of the tumor microenvironment may determine the free ISG15/ ISGylation action.

Knowing the integration of the modulation and functionality of ISG15 and the elements of the tumor microenvironment will enable the identification of new therapeutic targets, biomarkers, and markers of tumor progression, sensitivity, or treatment resistance. For instance, the deregulation of ISG15 expression in various cancer types and its presence at the extracellular level suggests its potential use as a biomarker.

Furthermore, the effects reported for ISG15 indicate its potential as a therapeutic agent. For example, decreasing ISG15 in PDAC triggered PDL-1 downregulation and a more effective anti-PD1 immunotherapy (Burks et al., 2019), suggesting its potential as a therapeutic strategy.

Further research is required to understand the mechanisms of regulation and action of ISG15 in the tumor microenvironment. The modulation of the tumor microenvironment by ISG15 and its collaboration with other factors may influence the decision of cell programs on continuing proliferation, migration, invasion, senescence or death.

In conclusion, ISG15 is an interesting molecule because of its multifunctionality in vertebrate organisms, high modulation capacity by various factors, and central participation in cancer pathologies. The impact of ISG15 on the proteome, secretome, and transcriptome could be dependent on the cellular context. The high functional complexity requires elucidation in specific contexts. Nevertheless, the studies suggest that ISG15 and ISGylation have a central role in the cytoskeleton dynamic and tumor microenvironment. 


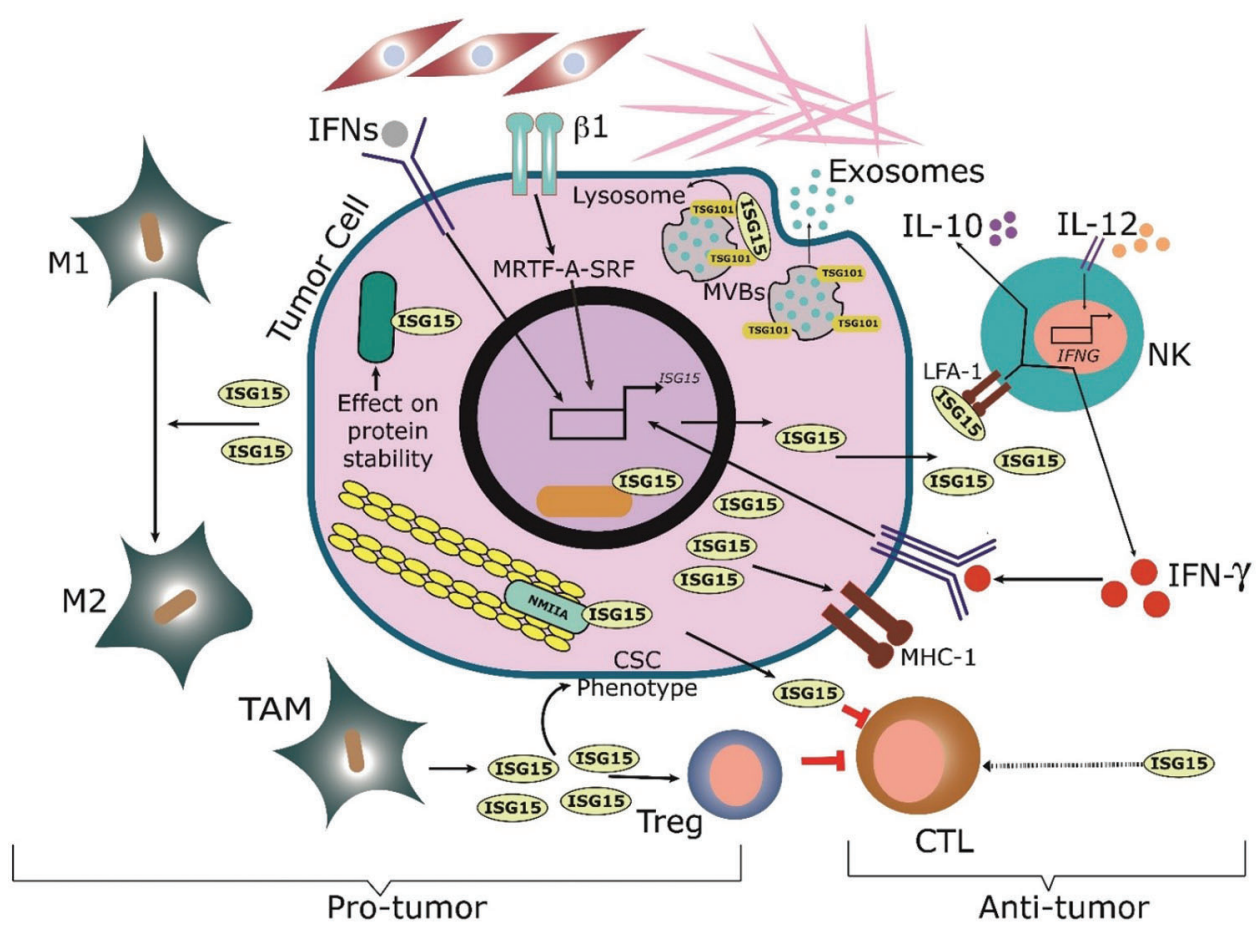

FIGURE 2. Free ISG15 and ISGylation in the cytoskeleton dynamic and tumor microenvironment. ISG15 and ISGylation have a pro-tumor and anti-tumor role. ISG15 induces the conversion from the M1 phenotype (pro-inflammatory and anti-tumor macrophages) to the M2 phenotype (anti-inflammatory and pro-tumoral macrophages). TAMs (tumor-associated macrophages) and tumor cells release ISG15 to the extracellular microenvironment. ISG15 can promote a CSC (cancer stem cell) phenotype. ISG15 inhibits CTLs (cytotoxic T lymphocytes) and activates Treg (regulatory T cells). Then, Treg cells inhibit CTLs. IFNs/IFN- $\gamma$ pathways and $\beta 1$ (integrin beta 1)dependent signaling induce ISG15 expression (long arrows). MRTF-A-SRF, a $\beta 1$-activated transcription complex, induces ISG15 expression (empty square = ISG15 promoter region). ISG15 is associated with the presence of MHC-1 (major histocompatibility complex class I). ISGylation promotes the MVBs (multivesicular bodies) degradation via lysosome, inhibiting exosome secretion. NMIIA (nonmuscle myosin IIA), an actin-associated protein, is modulated by ISGylation. Cytoplasmic proteins (green box) and nuclear proteins (orange box) are regulated by ISGylation. Natural killer (NK) cells have a receptor that recognizes ISG15 (LFA-1), promoting the secretion of interleukin 10 (IL-10) and IFN- $\gamma$. IL-12 induces the expression of IFN- $\gamma$ (IFNG gene. Empty Square $=$ IFNG promoter). Exogenous ISG15 seems to activate CTLs (dashed arrow). Cancer-associated fibroblasts and extracellular matrix are shown above.

Acknowledgement: I thank Dr. Marina Macías Silva for her valuable comments on the manuscript.

Author Contribution: The author confirms sole responsibility for the following: study conception and design, data collection, analysis and interpretation of results, and manuscript preparation.

Funding Statement: This research was supported by the School of Science and Technology (CCyT-2021-5) from the Autonomous University of Mexico City (UACM).

Conflicts of Interest: The authors declare that they have no conflicts of interest to report regarding the present study.

\section{References}

Betapudi V (2010). Myosin II motor proteins with different functions determine the fate of lamellipodia extension during cell spreading. PLoS One 5: e8560. DOI 10.1371/journal.pone.0008560.

Betapudi V, Licate LS, Egelhoff TT (2006). Distinct roles of nonmuscle myosin II isoforms in the regulation of MDA-MB-231 breast cancer cell spreading and migration. Cancer Research 66: 4725-4733. DOI 10.1158/0008-5472.CAN-05-4236.

Burks J, Fleury A, Livingston S, Smith JP (2019). ISG15 pathway knockdown reverses pancreatic cancer cell transformation and decreases murine pancreatic tumor growth via downregulation of PDL-1 expression. Cancer Immunology, Immunotherapy 68: 2029-2039. DOI 10.1007/s00262-019-02422-9.

Burks J, Reed RE, Desai SD (2014). ISGylation governs the oncogenic function of Ki-Ras in breast cancer. Oncogene 33: 794-803. DOI 10.1038/onc.2012.633.

Burks J, Reed RE, Desai SD (2015). Free ISG15 triggers an antitumor immune response against breast cancer: A new perspective. Oncotarget 6: 7221-7231. DOI 10.18632/oncotarget.3372.

Cai Y, Biais N, Giannone G, Tanase M, Jiang G et al. (2006). Nonmuscle myosin IIA-dependent force inhibits cell spreading and drives F-actin flow. Biophysical Journal 91: 3907-3920. DOI 10.1529/biophysj.106.084806.

Cerikan B, Shaheen R, Colo GP, Gläßer C, Hata S et al. (2016). Cellintrinsic adaptation arising from chronic ablation of a key rho GTPase regulator. Developmental Cell 39: 28-43. DOI 10.1016/j.devcel.2016.08.020.

Chen RH, Du Y, Han P, Wang HB, Liang FY et al. (2016). ISG15 predicts poor prognosis and promotes cancer stem cell phenotype in nasopharyngeal carcinoma. Oncotarget 7: 16910-16922. DOI 10.18632/oncotarget.7626.

Chen RH, Xiao ZW, Yan XQ, Han P, Liang FY et al. (2020). Tumor cell-secreted ISG15 promotes tumor cell migration and immune suppression by inducing the macrophage M2-like phenotype. Frontiers in Immunology 11: e1004561. DOI 10.3389/fimmu.2020.594775. 
Cruz-Ramos E, Macías-Silva M, Sandoval-Hernández A, TecalcoCruz AC (2019). Non-muscle myosin IIA is posttranslationally modified by interferon-stimulated gene 15 in breast cancer cells. International Journal of Biochemistry \& Cell Biology 107: 14-26. DOI 10.1016/j.biocel.2018.12.002.

Desai SD, Haas AL, Wood LM, Tsai YC, Pestka S et al. (2006). Elevated expression of ISG15 in tumor cells interferes with the ubiquitin/26S proteasome pathway. Cancer Research 66: 921-928. DOI 10.1158/0008-5472.CAN-05-1123.

Desai SD, Reed RE, Burks J, Wood LM, Pullikuth AK et al. (2012). ISG15 disrupts cytoskeletal architecture and promotes motility in human breast cancer cells. Experimental Biology and Medicine 237: 38-49. DOI 10.1258/ebm.2011.011236.

DiPersio CM, Longmate W (2017). Beyond adhesion: Emerging roles for integrins in control of the tumor microenvironment. F1000Research 6: 1612. DOI 10.12688/f1000research.

Ganesan M, Poluektova LY, Tuma DJ, Kharbanda KK, Osna NA (2016). Acetaldehyde disrupts interferon alpha signaling in hepatitis C virus-infected liver cells by up-regulating USP18. Alcoholism-Clinical and Experimental Research 40: 2329-2338. DOI 10.1111/acer.13226.

Hermann MR, Jakobson M, Colo GP, Rognoni E, Jakobson M et al. (2016). Integrins synergize to induce expression of the MRTF-A/SRF target gene ISG15 for promoting cancer cell invasion. Journal of Cell Science 97: 133. DOI 10.1242/ jcs. 177592.

Iglesias-Guimarais V, Ahrends T, de Vries E, Knobeloch KP, Volkov A et al. (2020). IFN-stimulated gene 15 is an alarmin that boosts the CTL response via an innate, NK cell-dependent route. Journal of Immunology 204: 2110-2121. DOI 10.4049/jimmunol.1901410.

Jeon YJ, Choi JS, Lee JY, Yu KR, Kim SM et al. (2009). ISG15 modification of filamin $\mathrm{B}$ negatively regulates the type I interferon-induced JNK signalling pathway. EMBO Reports 10: 374-380. DOI 10.1038/embor.2009.23.

Jorgovanovic D, Song M, Wang L, Zhang Y (2020). Roles of IFN- $\gamma$ in tumor progression and regression: A review. Biomarker Research 8: 1964. DOI 10.1186/s40364-020-00228-x.

Labani-Motlagh A, Ashja-Mahdavi M, Loskog A (2020). The tumor microenvironment: A milieu hindering and obstructing antitumor immune responses. Frontiers in Immunology 11: 1-22.

Lee JH, Bae JA, Lee JH, Seo YW, Kho DH et al. (2010). Glycoprotein $90 \mathrm{~K}$, downregulated in advanced colorectal cancer tissues, interacts with $\mathrm{CD} 9 / \mathrm{CD} 82$ and suppresses the $\mathrm{Wnt} / \beta$ catenin signal via ISGylation of $\beta$-catenin. Gut 59: 907-917. DOI 10.1136/gut.2009.194068.

Lei X, Lei Y, Li JK, Du WX, Li RG et al. (2020). Immune cells within the tumor microenvironment: Biological functions and roles in cancer immunotherapy. Cancer Letters 470: 126-133. DOI 10.1016/j.canlet.2019.11.009.

Li X, Wang J (2020). Mechanical tumor microenvironment and transduction: Cytoskeleton mediates cancer cell invasion and metastasis. International Journal of Biological Sciences 16: 2014-2028. DOI 10.7150/ijbs.44943.

Mao H, Wang M, Cao B, Zhou H, Zhang Z et al. (2016). Interferonstimulated gene 15 induces cancer cell death by suppressing the NF- $\mathrm{BB}$ signaling pathway. Oncotarget 7: 70143-70151. DOI 10.18632/oncotarget.12160.
Padovan E, Terracciano L, Certa U, Jacobs B, Reschner A et al. (2002). Interferon stimulated gene 15 constitutively produced by melanoma cells induces E-cadherin expression on human dendritic cells. Cancer Research 62: 3453-3458.

Sainz B, Martín B, Tatari M, Heeschen C, Guerra S (2014). ISG15 is a critical microenvironmental factor for pancreatic cancer stem cells. Cancer Research 74: 7309-7320. DOI 10.1158/00085472.CAN-14-1354.

Schoggins JW (2019). Interferon-stimulated genes: What do they all do? Annual Review of Virology 6: 567-584. DOI 10.1146/ annurev-virology-092818-015756.

Shi H-X, Yang K, Liu X, Liu XY, Wei B et al. (2010). Positive regulation of interferon regulatory factor 3 activation by Herc5 via ISG15 modification. Molecular and Cellular Biology 30: 2424-2436. DOI 10.1128/MCB.01466-09.

Swaim CD, Canadeo LA, Monte KJ, Khanna S, Lenschow DJ et al. (2020). Modulation of extracellular ISG15 signaling by pathogens and viral effector proteins. Cell Reports 31: 107772. DOI 10.1016/j.celrep.2020.107772.

Swaim CD, Scott AF, Canadeo LA, Huibregtse JM (2017). Extracellular ISG15 signals cytokine secretion through the LFA-1 integrin receptor. Molecular Cell 68: 581-590.e5. DOI 10.1016/j.molcel.2017.10.003.

Tang K, Xin Y, Li K, Chen X, Tan Y (2021). Cell cytoskeleton and stiffness are mechanical indicators of organotropism in breast cancer. Biology (Basel) 10: 259.

Tecalco-Cruz AC (2020). Molecular pathways of interferon-stimulated gene 15: Implications in Cancer. Current Protein \& Peptide Science 22: 19-28. DOI 10.2174/1389203721999201208200747.

Tecalco-Cruz AC, Cortés-González CC, Cruz-Ramos E, RamírezJarquín JO, Romero-Mandujano AK et al. (2019). Interplay between interferon-stimulated gene 15/ISGylation and interferon gamma signaling in breast cancer cells. Cellular Signalling 54: 91-101. DOI 10.1016/j.cellsig.2018.11.021.

Villarroya-Beltri C, Baixauli F, Mittelbrunn M, Fernández-Delgado I, Torralba D et al. (2016). ISGylation controls exosome secretion by promoting lysosomal degradation of MVB proteins. Nature Communications 7: 1303. DOI 10.1038/ ncomms 13588 .

Yeung TL, Tsai CC, Leung CS, Yeung CLA, Thompson MS et al. (2018). ISG15 promotes ERK1 ISGylation, CD8+ T cell activation and suppresses ovarian cancer progression. Cancers (Basel) 10: 464. DOI 10.3390/cancers10120464.

Yuan H, Zhou W, Yang Y, Xue L, Liu L et al. (2018). ISG15 promotes esophageal squamous cell carcinoma tumorigenesis via c-MET/Fyn/ $\beta$-catenin signaling pathway. Experimental Cell Research 367: 47-55. DOI 10.1016/j.yexcr.2018.03.017.

Zhang DX, Vu LT, Ismail NN, Le MTN, Grimson A (2021). Landscape of extracellular vesicles in the tumour microenvironment: Interactions with stromal cells and with non-cell components, and impacts on metabolic reprogramming, horizontal transfer of neoplastic traits, and the emergence of therapeutic resistance. Seminars in Cancer Biology 74: 24-44. DOI 10.1016/j.semcancer.2021.01.007.

Zhou MJ, Chen FZ, Chen HC, Wan XX, Zhou X et al. (2017). ISG15 inhibits cancer cell growth and promotes apoptosis. International Journal of Molecular Medicine 39: 446-452. DOI 10.3892/ijmm.2016.2845. 